\title{
De novo CD5-positive diffuse large B-cell lymphomas show high specificity for cyclin D2 expression
}

\author{
Takuro Igawa' ${ }^{1}$, Yasuharu Sato ${ }^{*}$, Katsuyoshi Takata', Noriko Iwaki ${ }^{1}$, Takehiro Tanaka², Naoko Asano ${ }^{3}$, \\ Yoshinobu Maeda ${ }^{4}$, Yorihisa Orita ${ }^{5}$, Naoya Nakamura ${ }^{6}$, Shigeo Nakamura ${ }^{7}$ and Tadashi Yoshino ${ }^{1}$
}

\begin{abstract}
D cyclins positively regulate the cell cycle and mediate the pathogenesis of some lymphomas. Cyclin D1 overexpression is the hallmark of mantle cell lymphoma, whereas cyclins D2 and D3 are reportedly not as specific to certain lymphomas as cyclin D1. In this study, cyclin D2 was found to be overexpressed in $98 \%$ of de novo CD5-positive diffuse large B-cell lymphomas (DLBCLs) (50/51) and in 28\% of CD5-negative DLBCLs (14/51). A statistically significant difference was observed between these two groups $(p<0.0001)$. In contrast, no statistical difference was found in the cyclin D3 expression between CD5-positive (18/51) and CD5-negative (24/51) DLBCLs $(p=0.23)$. Based on these findings, cyclin D2 is therefore considered to be closely associated with de novo CD5-positive DLBCLs. This insight may be useful for overcoming the inferior survival of this aggressive lymphoma. Virtual slides: The virtual slide(s) for this article can be found here: http://www.diagnosticpathology.diagnomx.eu/ vs/1382856320966453
\end{abstract}

Keywords: Cyclin D2, CD5, Diffuse large B-cell lymphoma

\section{Background}

D-type cyclins (D1, D2, and D3) positively regulate the cell cycle and mediate the pathogenesis of some lymphomas [1]. The overexpresison of cyclin D1 due to translocation $\mathrm{t}(11 ; 14)(\mathrm{q} 13 ; \mathrm{q} 32)$ serves as a hallmark for the diagnosis of mantle cell lymphoma [1]. Recently, a rare type of cyclin D1-negative mantle cell lymphoma, which overexpresses cyclins D2 or D3, was identified [2]. Cyclins D2 and D3 are also detected in various lymphomas, but they have not been shown to be closely associated with any particular subtype of lymphoma [3].

We recently demonstrated cyclin D2 to be overexpressed in the proliferation centers of chronic lymphocytic leukemia/small lymphocytic lymphoma (CLL/SLL) [4]. CLL/SLL is considered to be a disease caused by the accumulation of CD5-positive B lymphocytes, and proliferating tumor cells are located in the proliferation

\footnotetext{
* Correspondence: satou-y@cc.okayama-u.ac.jp

'Department of Pathology, Okayama University Graduate School of Medicine, Dentistry and Pharmaceutical Sciences, 2-5-1 Shikata-cho, Okayama 700-8558, Japan

Full list of author information is available at the end of the article
}

centers of lymph nodes [5]. The proliferating cells are composed of paraimmunoblastic cells and most of them are in the cell cycle, expressing Ki-67 antigen [5]. These findings suggest that de novo CD5-positive diffuse large B-cell lymphomas ( $\mathrm{CD}^{+}$DLBCLs) share the characteristics of actively proliferating cells in CLL/SLL.

De novo $\mathrm{CD}^{+}$DLBCLs account for approximately $10 \%$ of all DLBCL cases, and they are characterized by a female predominance, a higher age at diagnosis, and a high degree of central nervous system relapse [6]. De novo $\mathrm{CD}^{+}$ DLBCLs are also known to show a significantly poorer survival outcome than CD5-negative DLBCLs (CD5 ${ }^{-}$DLBCLs) under both cyclophosphamide, doxorubicin, vincristine, and prednisone (CHOP) and rituximab-CHOP (R-CHOP) therapy [6,7].

According to these previous data, we sought to clarify the expression patterns of cyclins D2 and D3 in de novo CD5 ${ }^{+}$DLBCLs.

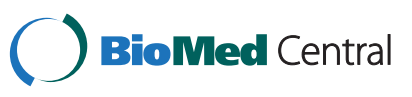

(c) 2013 Igawa et al.; licensee BioMed Central Ltd. This is an Open Access article distributed under the terms of the Creative Commons Attribution License (http://creativecommons.org/licenses/by/2.0), which permits unrestricted use, distribution, and reproduction in any medium, provided the original work is properly cited. 
Table 1 Cyclins D2 and D3 expression in de novo $\mathrm{CD}^{+}$ and $\mathrm{CD}^{-}$DLBCLs

\begin{tabular}{lccc}
\hline & De novo CD5 ${ }^{+}$DLBCLs & CD5 $^{-}$DLBCLs & P \\
\hline Cyclin D2 & $50 / 51(98 \%)$ & $14 / 51(28 \%)$ & $P<0.0001$ \\
Cyclin D3 & $18 / 51(35 \%)$ & $24 / 51(47 \%)$ & $P=0.23$ \\
\hline
\end{tabular}

\section{Methods}

\section{Case selection}

We studied 51 Japanese patients with de novo $\mathrm{CD}^{+}$ DLBCLs diagnosed between 1998 and 2011 at Okayama University, Tokai University, and Nagoya University. The patients included 26 males and 25 females between 32 and 90 years of age (median age 68 years). The examined tissue specimens were obtained from 32 lymph nodes and 19 extranodal sites. The CD5 antigen expression was examined by means of immunohistochemistry of paraffin sections and/or flow cytometry. All samples were immunohistochemically confirmed to be cyclin D1 and sox11 negative [2]. Any samples with a history of other lymphoproliferative disorders were excluded from the study.

As a control group, samples taken from 51 patients with CD5 $5^{-}$DLBCLs diagnosed between 1997 and 2011 at Okayama University were also examined. The patients included 27 males and 24 females between 23 and 89 years of age (median age 68 years). The examined tissue specimens were obtained from 42 lymph nodes and 9 extranodal sites. In all cases, the CD5 antigen expression was examined by both immunohistochemistry and flow cytometry.
Table 2 Previous reports on cyclins D2 and D3 expression by immunohistochemistry in DLBCLs

\begin{tabular}{lccccc}
\hline Reference (reference no.) & $\begin{array}{c}\text { No. of } \\
\text { patients }\end{array}$ & $\begin{array}{c}\text { Cut-off } \\
\text { line (\%) }\end{array}$ & \multicolumn{2}{c}{ Positive cases (\%) } \\
\hline Hans CP, et al., 2004 [8] & 152 & 30 & 13 & Cyclin D3 \\
\hline Hans CP, et al., 2005 [9] & 200 & 30 & 19 & 62 \\
Amen F, et al., 2007 [10] & 80 & 10 & 25 & ND \\
Metcalf RA, et al., 2010 [3] & 194 & 20 & 49 & 20 \\
Present study CD5 ${ }^{+}$DLBCLs & 51 & 20 & 98 & 35 \\
\multicolumn{1}{c}{ CD5 ${ }^{-}$DLBCLs } & 51 & 20 & 28 & 47 \\
\hline
\end{tabular}

$\mathrm{ND}$, not done.

\section{Histological examination and immunohistochemistry}

The tissue samples were fixed in $10 \%$ formalin and embedded in paraffin. The sections (4- $\mu \mathrm{m}$ thick) were stained with H\&E. Immunohistochemistry was performed on the paraffin-fixed sections using an automated Bond-max stainer (Leica Biosystems, Melbourne, Australia) and anti-cyclin D2 (polyclonal; 1:150; Proteintech Group Inc., Chicago, IL, USA) and anti-cyclin D3 (DCS-22; 1:10; Progen Biotechnik GmbH, Heidelberg, Germany) antibodies. Based on previous studies, a sample was considered to be positive if $\geq 20 \%$ of the tumor cells were stained [3]. Faint cytoplasmic staining for cyclin D2 without corresponding nuclear staining was not considered positive.

\section{Statistical analysis}

The correlations between the 2 groups were examined by a chi-square analysis. All statistical analyses were carried out with the SPSS software program (version 14.0, SPSS Inc., Chicago, USA).

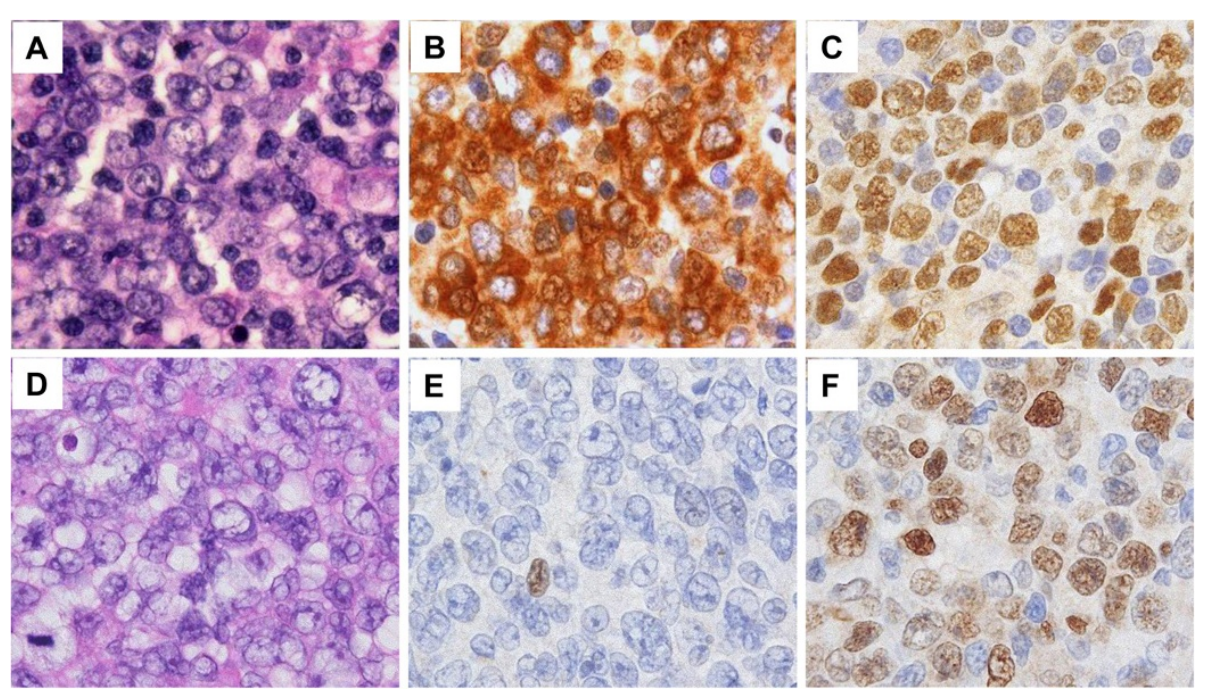

Figure 1 Cyclins D2 and D3 expression in de novo CD5-positive and CD5-negative DLBCLs. Cyclin D2 expression in de novo CD5-positive $(\mathbf{A}, \mathbf{B}$, and $\mathbf{C})$ and CD5-negative (D, E, and $\mathbf{F})$ DLBCLs, H\&E staining $(\mathbf{A} \& \mathbf{D})$, cyclin D2 staining (B \& E), and cyclin D3 staining $(\mathbf{C} \& \mathbf{F})(\times 400)$. Cyclin D2 staining was frequently localized to both the nucleus and the cytoplasm in the de novo CD5-positive DLBCLs (B). Cyclin D3 showed a crisp nuclear staining pattern in both CD5-positive and negative DLBCLS (C \& F). 


\section{Results and discussion}

In this study, our data showed that cyclin D2 was overexpressed in $98 \%$ of de novo $\mathrm{CD}^{+}$DLBCLs (50/51) and in $28 \%$ of $\mathrm{CD}^{-}$DLBCLs (14/51) (Table 1, Figure 1). A statistically significant difference was observed between these two groups $(\mathrm{p}<0.0001)$. In contrast, no statistical difference was found in the cyclin D3 expression between CD5-positive (18/51) and CD5-negative (24/51) DLBCLs $(\mathrm{p}=0.23)$ (Table 1, Figure 1). Since de novo CD5 ${ }^{+}$DLBCLs are typically immunohistochemically negative for cyclin D1 [6], these findings therefore indicate that cyclin D2 is closely associated with de novo $\mathrm{CD}^{+}$DLBCLs.

Previous studies have examined the cyclins D2 and D3 expression in DLBCLs by immunohistochemistry (Table 2) [3,8-10]. Although the rates of cyclins D2 and D3 positive cases were highly variable in these studies, our findings suggest that cyclin D2-positive DLBCLs comprise the majority of de novo $\mathrm{CD} 5^{+}$DLBCLs.

Several studies have shown cyclin D2 to be an independent indicator of an inferior survival in DLBCLs [8-10]. In addition, Lossos et al. identified CCND2 to be the best predictor of an inferior survival in DLBCLs among the 36 genes associated with their prognosis based on quantitative RT-PCR [11]. These data suggest that cyclin D2 may be associated with the inferior survival observed in de novo $\mathrm{CD}^{+}$DLBCLs. The current gold standard therapy has evolved to include rituximab, and the outcome of DLBCLs has been reported to have dramatically improved [12]. However, both CCND2 and CD5 have been demonstrated to still be a good indicator of the inferior survival in DLBCLs, even in the era of R-CHOP $[7,12]$. This fact also suggests an association between cyclin D2 and the poor survival in de novo $\mathrm{CD}^{+}$DLBCLs. Similar to de novo $\mathrm{CD}^{+}$DLBCLs, cyclin D2-positive CD5 ${ }^{-}$DLBCLs may play a role in the negative prognostic impact of cyclin D2 in DLBCLs. In the current study, however, whether or not cyclin D2-positive CD $5^{-}$DLBCLs show a poor survival was not examined. As a result, the prognostic impact of this population therefore still needs to be investigated.

\section{Conclusions}

Cyclin D2 is closely associated with de novo $\mathrm{CD} 5^{+}$DLBCLs. This insight might be useful for making treatment strategies and improving the survival of this aggressive lymphoma, since cyclin D2 expression is controlled by multiple signaling pathways, such as the NFkB-related pathways [13].

\section{Consent}

Informed consent was obtained from the patient for publication of this report and any accompanying images.

\section{Abbreviations}

DLBCL: Diffuse large B-cell lymphoma; CLL/SLL: Chronic lymphocytic leukemia/small lymphocytic lymphoma; CHOP: Cyclophosphamide, doxorubicin, vincristine, and prednisone; R-CHOP: Rituximab with cyclophosphamide, doxorubicin, vincristine, and prednisone; RT-PCR: Reverse transcription polymerase chain reaction; NFkB: Nuclear factor kappa beta.

\section{Competing interests}

The authors declare that they have no competing interest.

\section{Authors' contributions}

Conceived and designed the experiments: YS, TI. Performed the experiments: TI, YS. Analyzed the data: YS, TI, TY, KT, NI, TT, YM, NA, YO. Contributed reagents/materials/analysis tools: NN, SN, YM. Wrote the paper: TI, YS, TY. All authors read and approved the final manuscript.

\section{Author details}

'Department of Pathology, Okayama University Graduate School of Medicine, Dentistry and Pharmaceutical Sciences, 2-5-1 Shikata-cho, Okayama 700-8558, Japan. ${ }^{2}$ Department of Pathology, Okayama University Hospital, Okayama, Japan. ${ }^{3}$ Department of Clinical Laboratory, Nagoya University Hospital, Nagoya, Japan. ${ }^{4}$ Department of Hematology and Oncology, Okayama University Graduate School of Medicine, Dentistry and Pharmaceutical Sciences, Okayama, Japan. ${ }^{5}$ Department of Otolaryngology, Head and Neck Surgery, Okayama University Graduate School of Medicine, Dentistry and Pharmaceutical Science, Okayama, Japan. ${ }^{6}$ Department of Pathology, Tokai University School of Medicine, Isehara, Japan. ${ }^{7}$ Department of Pathology, Nagoya University Hospital, Nagoya, Japan.

Received: 14 April 2013 Accepted: 30 April 2013

Published: 15 May 2013

\section{References}

1. Yatabe $Y$, Suzuki R, Tobinai $K$, et al: Significance of cyclin D1 overexpression for the diagnosis of mantle cell lymphoma: a clinicopathologic comparison of cyclin D1-positive MCL and cyclin D1-negative MCL-like B-cell lymphoma. Blood 2000, 95:2253-61.

2. Mozos A, Royo C, Hartmann E, et al: SOX11 expression is highly specific for mantle cell lymphoma and identifies the cyclin D1-negative subtype. Haematologica 2010, 95(9):1620.

3. Metcalf RA, Zhao SC, Anderson MW, et al: Characterization of D-cyclin proteins in hematolymphoid neoplasms: lack of specificity of cyclin-D2 and D3 expression in lymphoma subtypes. Mod Pathol 2010, 23:420-33.

4. Igawa T, Sato Y, Takata K, et al: Cyclin D2 is overexpressed in proliferation centers of chronic lymphocytic leukemia/small lymphocytic lymphoma. Cancer Sci 2011, 102:2103-7.

5. Chiorazzi N, Rai KR, Ferrarini M: Mechanisms of disease: Chronic lymphocytic leukemia. N Engl J Med 2005, 352:804-15.

6. Yamaguchi M, Seto M, Okamoto M, et al: De novo CD5(+) diffuse large B-cell lymphoma: a clinicopathologic study of 109 patients. Blood 2002, 99:815-21.

7. Miyazaki K, Yamaguchi M, Suzuki R, et al: CD5-positive diffuse large B-cell lymphoma: a retrospective study in 337 patients treated by chemotherapy with or without rituximab. Ann Oncol 2011, 22:1601-7.

8. Hans CP, Weisenburger DD, Greiner TC, et al: Confirmation of the molecular classification of diffuse large B-cell lymphoma by immunohistochemistry using a tissue microarray. Blood 2004, 103:275-82.

9. Hans CP, Weisenburger DD, Greiner TC, et al: Expression of PKC-beta or cyclin D2 predicts for inferior survival in diffuse large B-cell lymphoma. Mod Pathol 2005, 18:1377-84.

10. Amen F, Horncastle D, Elderfield $K$, et al: Absence of cyclin-D2 and Bcl-2 expression within the germinal centre type of diffuse large B-cell lymphoma identifies a very good prognostic subgroup of patients. Histopathology 2007, 51:70-9.

11. Lossos IS, Czerwinski DK, Alizadeh AA, et al: Prediction of survival in diffuse large-B-cell lymphoma based on the expression of six genes. N Engl J Med 2004, 350:1828-37.

12. Malumbres R, Chen J, Tibshirani R, et al: Paraffin-based 6-gene model predicts outcome in diffuse large B-cell lymphoma patients treated with R-CHOP. Blood 2008, 111:5509-14.

13. Chiles TC: Regulation and function of cyclin D2 in B lymphocyte subsets. $J$ Immunol 2004, 173:2901-7.

doi:10.1186/1746-1596-8-81

Cite this article as: Igawa et al:: De novo CD5-positive diffuse large B-cell lymphomas show high specificity for cyclin D2 expression. Diagnostic Pathology 2013 8:81. 